\title{
Detection of CS emission towards Cygnus OB2 No. 12
}

\author{
F. Scappini ${ }^{1}$, C. Cecchi-Pestellini ${ }^{2}$, S. Casu ${ }^{2}$, and M. Olberg ${ }^{3}$ \\ 1 Istituto per lo Studio dei Materiali Nanostrutturati del C.N.R., via P. Gobetti 101, 40129 Bologna, Italy \\ e-mail: F.Scappini@bo.ismn.cnr.it \\ 2 INAF - Osservatorio Astronomico di Cagliari, Strada n.54, Loc. Poggio dei Pini, 09012 Capoterra (CA), Italy \\ e-mail: [ccp;silvia]@ca.astro.it \\ 3 Onsala Space Observatory, 43900 Onsala, Sweden \\ e-mail: michael.olberg@chalmers.se
}

Received 29 June 2006 / Accepted 27 January 2007

\begin{abstract}
Aims. The CS $(J=2-1)$ line was searched at locations with brightest ${ }^{13} \mathrm{CO}(J=1-0)$ emissions in the region in front of the star Cyg OB2 No. 12.

Methods. The observations were made with the 20-m millimeter wave telescope at Onsala using a 1024-channel autocorrelator with a resolution of $50 \mathrm{kHz}\left(0.14 \mathrm{~km} \mathrm{~s}^{-1}\right)$.

Results. The line was detected only in one position (core $B$ ) with main beam brightness temperature $T_{\mathrm{MB}}=0.15(2) \mathrm{K}$ at $v_{\mathrm{LSR}}=$ 11.4(1) $\mathrm{km} \mathrm{s}^{-1}$.

Conclusions. The line excitation analysis suggests that core $B$ could be a Bok globule in consideration of its morphology, chemistry and physical conditions.
\end{abstract}

Key words. ISM: clouds - ISM: lines and bands - ISM: molecules

\section{Introduction}

The chemistry of diffuse interstellar clouds has been traditionally considered simpler to understand than that of dense regions, as their chemistry and thermodynamics would be directly linked to the local density of the diffuse interstellar radiation. However, despite the apparent simplicity and the extensive set of gas-phase chemical models developed in about three decades remarkable discrepancies exist between models and observations. Besides the long-standing problem posed by the large concentrations of $\mathrm{CH}^{+}$, a number of molecular species, which should not exist in detectable amounts according to gas-phase ion-chemistry models, have been recently observed along diffuse lines of sight (Lucas \& Liszt 2000; Liszt \& Lucas 2001; Lucas \& Liszt 2002; Liszt \& Lucas 2002). Further uncertainty on the nature of diffuse-cloud chemistry is generated by the discovery, in these regions, of concentrations of the $\mathrm{H}_{3}^{+}$molecular ion (McCall et al. 1998) as large as those observed in dark clouds (Geballe \& Oka 1996). Such abundances appear inconsistent with model predictions based on a high removal rate via electronic dissociative recombination (Kokooline et al. 2001; McCall et al. 2003). $\mathrm{H}_{3}^{+}$is now being easily observed in the diffuse interstellar medium (McCall et al. 2002), but the uncertainty in its chemistry prevents this important species to be a useful probe of the conditions of diffuse clouds. Since the initial suggestion of McCall et al. (1998), who proposed that $\mathrm{H}_{3}^{+}$ forms in extended layers of very diffuse gas, a few other models were put forward in which $\mathrm{H}_{3}^{+}$chemistry has been discussed with morphology and physical properties of diffuse interstellar clouds (Cecchi-Pestellini \& Dalgarno 2000; Gredel et al. 2001; McCall et al. 2003; Le Petit et al. 2004). The said models have produced quite different results, in particular on the derived cloud structure.

To contribute data on the morphology, physics and chemical composition of diffuse clouds we studied the regions towards the stars Cyg OB2 No. 5 and No. 12 (Scappini et al. 2000, 2002; Casu et al. 2005). The observations provided evidence of two molecular structures at $7.3 \mathrm{~km} \mathrm{~s}^{-1}$, and $12.3 \mathrm{~km} \mathrm{~s}^{-1}$, both covering the portion of sky in fronts of the two stars. The gas layers at $12.3 \mathrm{~km} \mathrm{~s}^{-1}$ presents emission peaks with a core-halo morphology: core $A$ (close to Cyg OB2 No. 12, Scappini et al. 2000) and core $C$ (close to Cyg. OB2 No. 5, Casu et al. 2005). Only one core is evident in the $7.3 \mathrm{~km} \mathrm{~s}^{-1}$ kinematic structure (core $D$, Casu et al. 2005). All of these diffuse clumps have a small area and are embedded in a very low column density environment. The presence of gas condensations of distinct velocities had been also inferred from $\mathrm{C}_{2}$ absorption measurements (Gredel \& Münch 1994; Gredel et al. 2001). Such structures might be a rather common feature in the interstellar medium as suggested by the serendipitous detections of Heithausen (2002).

The two regions traced by the velocity fields at $7.3 \mathrm{~km} \mathrm{~s}^{-1}$ and $12.3 \mathrm{~km} \mathrm{~s}^{-1}$ overlap a brighter third region at $11.1 \mathrm{~km} \mathrm{~s}^{-1}$ (core B, cf. Scappini et al. 2002), north-west Cyg OB2 No. 12 in the direction towards Cyg OB2 No. 5, offset the lines of sight of both stars. Core $B$ contour map is roughly spherical with line intensity decreasing with incressing distance from the emission peak. On the contrary, cores $A$ and $C$ are part of a much wider clumpy region with irregular shape extending about 300 arcsec in both declination and RA. In core A Scappini et al. (2000) reported the detection of $\mathrm{HCO}^{+}$, through the emission of the $(1-0)$ rotational transition at $89 \mathrm{GHz}$, with a $v_{\mathrm{LSR}}=12.3 \mathrm{~km} \mathrm{~s}^{-1}$, in a location offset the line of sight to the star Cyg OB2 No. 12. 
The weak observed main beam brightness temperature, $\sim 0.09 \mathrm{~K}$, is consistent with previous detections of $\mathrm{HCO}^{+}$along diffuse, or marginally translucent, lines of sight (Lucas \& Liszt 1996). Unfortunately, $\mathrm{HCO}^{+}$emissions have not been measured in cores $B$ and $C^{1}$.

In this paper, we present observations of the CS $(J=$ 2-1) emission from cores $A$ and $B$. In diffuse clouds CS shares with CO an uncertain origin. Lucas \& Liszt (2002) showed that both species are well reproduced whenever the observed concentrations of their precursors, $\mathrm{HCO}^{+}$and $\mathrm{HCS}^{+}$, are inserted into standard chemical models of diffuse clouds. However, the same models fail to explain the presence of precursors.

CS absorption was sought in the $\zeta$ Oph spectrum taken with Copernicus but not detected and only an upper limit was derived (Snow 1976). A search of CS absorption corresponding to the $A-X(0,0)$ electronic transition near $257.6 \mathrm{~nm}$ towards various stars has been unsuccessful. Like CO, CS dissociates primarily through discrete absorptions into predissociating states, the strongest of these bands being the $C-X$ close to $140.0 \mathrm{~nm}$. Smith et al. (2001) reported the tentative identification of this CS band in the interstellar spectra of $\zeta$ Per, $\chi$ Oph, and HD 154368. All these detections have not yet been confirmed. The CS molecule has been detected at longer wavelengths through mm absorption lines in a number of diffuse clouds (Lucas \& Liszt 2002). Drdla et al. (1989) reported very weak CS $(J=2-1)$ emissions towards several objects with low-extinction lines of sight $\left(A_{\mathrm{V}} \lesssim 1 \mathrm{mag}\right)$, including $\zeta$ Oph. However, Kopp et al. (1996), and more recently Lucas \& Liszt (2002), failed to confirm the detection of the CS $(J=2-1)$ emission along the line of sight towards $\zeta$ Oph. Gredel et al. (1994) presented emission line data of $\mathrm{CS}$, together with $\mathrm{CN}$ and $\mathrm{HCO}^{+}$in diffuse and translucent clouds. Detections were obtained only in the case of rather strong ${ }^{13} \mathrm{CO} /{ }^{12} \mathrm{CO}$ intensity ratio. We conclude that $\mathrm{CS}$ emission has probably never been observed from diffuse clouds while it is commonly detected from translucent clouds and cirrus cores (e.g. Turner 1996).

In Sect. 2 observational details are presented. In Sect. 3, we discuss results and implications of the observations. Section 4 contains our conclusions.

\section{Observations}

The observations were made in May 2004 with the 20-m millimeter wave telescope at Onsala using a 1024-channel autocorrelator with a resolution of $50 \mathrm{kHz}\left(0.14 \mathrm{~km} \mathrm{~s}^{-1}\right)$. The single-side band tuned SIS receiver was operated in the frequency switching mode with an offset of $\pm 10 \mathrm{MHz}$. A chopper-wheel calibration technique was used. The pointing accuracy, checked every $3 \mathrm{~h}$, was found to be better than 4 arcsec rms on all occasions.

We searched for the CS $(J=2-1)$ emission, at $98 \mathrm{GHz}$, at the locations that showed the brightest ${ }^{13} \mathrm{CO}$ emission in the diffuse cloud in front of Cyg OB2 No. 12, i.e. the centres of cores $A$ and $B$ (Scappini et al. 2002). We detected CS emission only in core $B$ with a peak main beam brightness temperature $T_{\mathrm{MB}}=0.15(2) \mathrm{K}$ at $v_{\mathrm{LSR}}=11.4(1) \mathrm{km} \mathrm{s}^{-1}$, line width of $1.2(4) \mathrm{km} \mathrm{s}^{-1}$, and $\mathrm{rms}=23(2) \mathrm{mK}$ after $4.3 \mathrm{~h}$ integration time, see Fig. 1.

The non-detection in core $A$ provided a rms $=12(2) \mathrm{mK}$ after $5 \mathrm{~h}$ integration time. Previously, Scappini et al. (2000) have

\footnotetext{
1 Indeed, $\mathrm{HCO}^{+}$was searched for during the observational campaign reported in Scappini et al. (2002). However, the very high system temperature prevented any signficant measurement at the $\mathrm{HCO}^{+}$emision frequency.
}

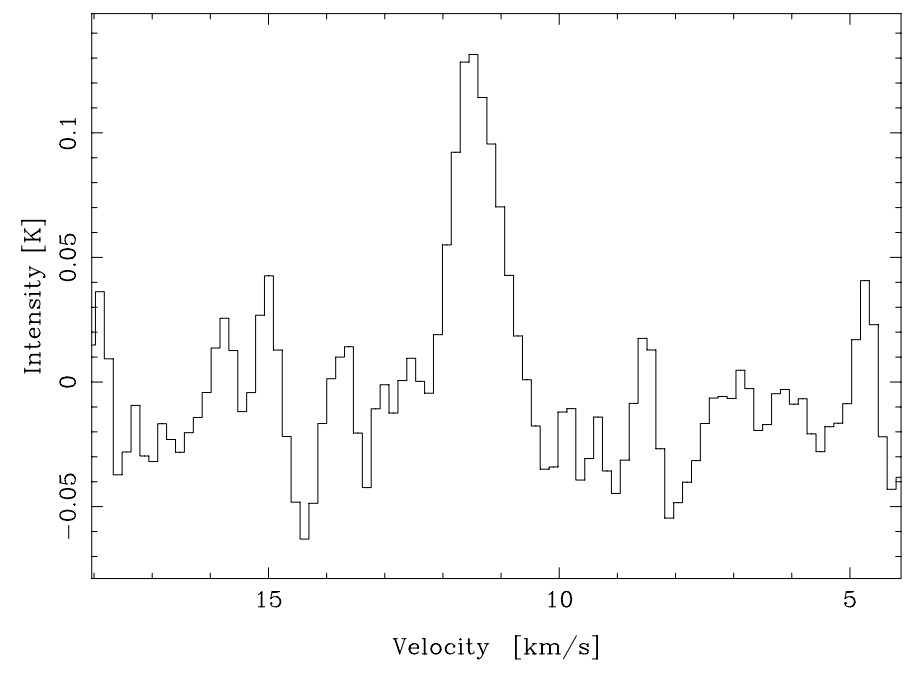

Fig. 1. The CS $(J=2-1)$ emission line detected in the core $B$ at $v_{\mathrm{LSR}}=$ 11.4(1) $\mathrm{km} \mathrm{s}^{-1}$.

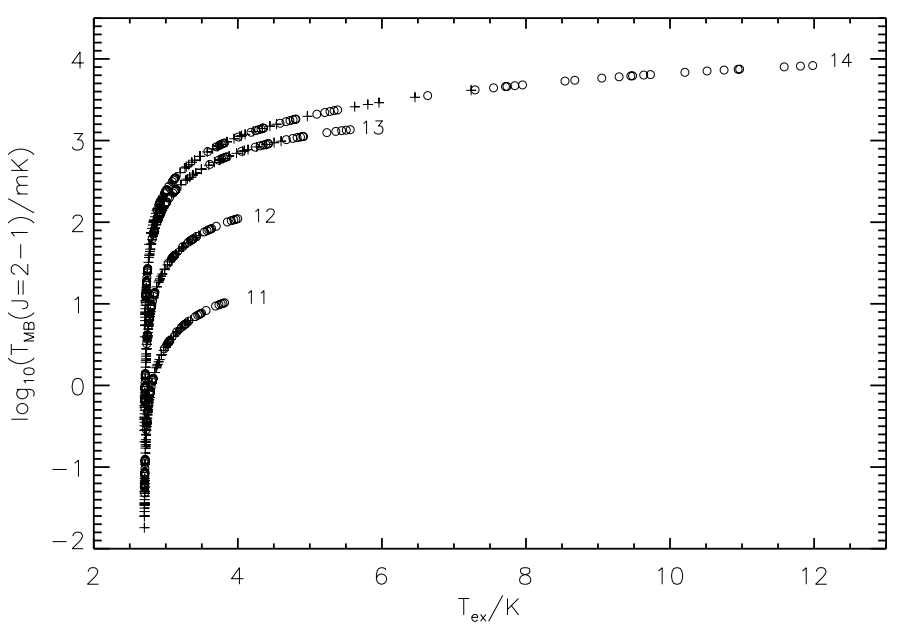

Fig. 2. Mean-beam brightness temperature $\left(T_{\mathrm{MB}}\right)$ of the $\mathrm{CS}(J=$ $2-1)$ line vs. the corresponding excitation temperature $\left(T_{\text {ex }}\right)$ for several assumptions on the chemical and physical condition of the emitting gas. $x_{\mathrm{e}}=10^{-5}$ crosses; $x_{\mathrm{e}}=10^{-4}$ open circles. Labels refer to $\log _{10}\left(N_{\mathrm{CS}} / \Delta v\right) / \mathrm{cm}^{-2}\left(\mathrm{~km} \mathrm{~s}^{-1}\right)^{-1}$.

searched for the CS $(J=3-2)$ emission in core $A$ and failed to detect it to a rms limit of 20(2) $\mathrm{mK}$.

\section{Discussion}

CS $(J=2-1)$ emission is not generally observed along diffuse lines of sight (cf. Lucas \& Liszt 2002), while it is easily detected in translucent regions (Turner 1996) and Bok globules (Henning \& Launhardt 1998). Thus, the present observations, when incorporated in the general observational scenario, might provide evidence that cores $A$ and $B$ present different excitation conditions, respectively.

In order to model the excitation conditions of CS, statistical equilibrium calculations are used. We solved the following set 

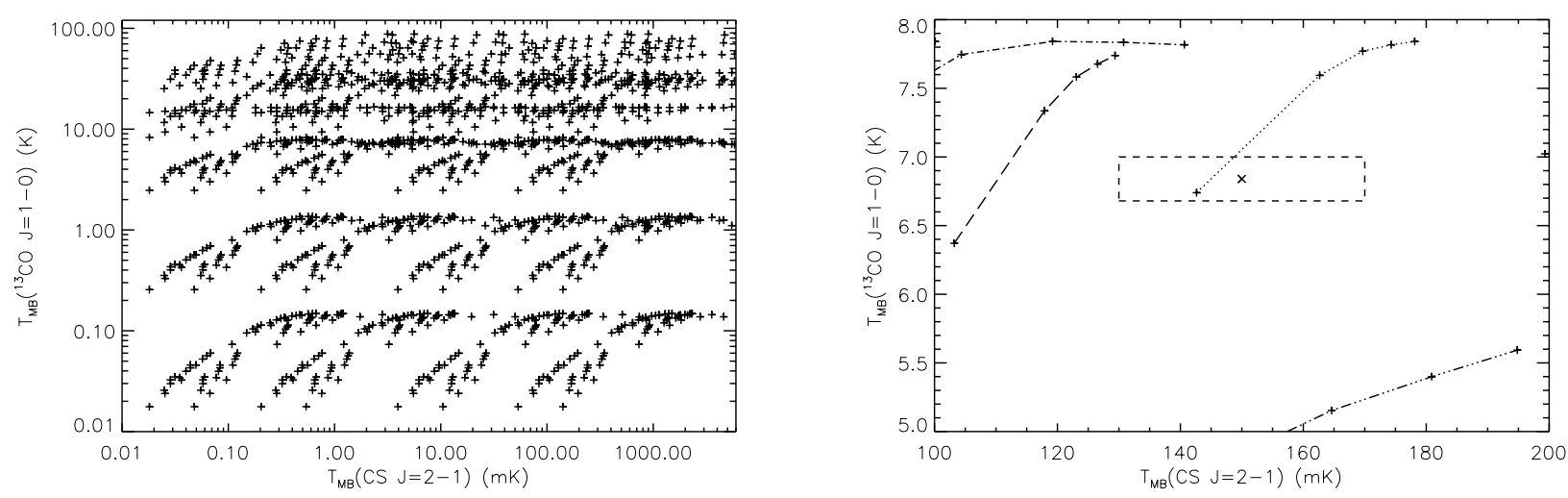

Fig. 3. Modelled mean-beam brightness temperatures of ${ }^{13} \mathrm{CO}(J=1-0)$ and $\mathrm{CS}(J=2-1)$. a) $n_{\mathrm{H}}=50-10000 \mathrm{~cm}^{-3}, T_{\mathrm{k}}=20-100 \mathrm{~K}$, $x_{\mathrm{e}}=10^{-5}-10^{-4}, N_{\mathrm{CS}} / \Delta v=10^{11}-10^{14} \mathrm{~cm}^{-2}\left(\mathrm{~km} \mathrm{~s}^{-1}\right)^{-1}$, and $\left.N_{13} \mathrm{CO} / \Delta v=10^{13}-10^{18} \mathrm{~cm}^{-2}\left(\mathrm{~km} \mathrm{~s}^{-1}\right)^{-1} ; \mathbf{b}\right)$ blow-up around the observational point (cross). The dashed box indicates the errors in the measurements. Dotted line: $n_{\mathrm{H}}=700 \mathrm{~cm}^{-3}, N_{\mathrm{CS}} / \Delta v=10^{13} \mathrm{~cm}^{-2}\left(\mathrm{~km} \mathrm{~s}^{-1}\right)^{-1}, N_{13} \mathrm{CO} / \Delta v=$ $10^{16} \mathrm{~cm}^{-2}\left(\mathrm{~km} \mathrm{~s}^{-1}\right)^{-1}, x_{\mathrm{e}}=10^{-4}$; dashed line: $n_{\mathrm{H}}=500 \mathrm{~cm}^{-3}, N_{\mathrm{CS}} / \Delta v=10^{13} \mathrm{~cm}^{-2}\left(\mathrm{~km} \mathrm{~s}^{-1}\right)^{-1}, N_{13} \mathrm{Co} / \Delta v=10^{16} \mathrm{~cm}^{-2}\left(\mathrm{~km} \mathrm{~s}^{-1}\right)^{-1}, x_{\mathrm{e}}=10^{-4} ;$ dashdotted line: $n_{\mathrm{H}}=1000 \mathrm{~cm}^{-3}, N_{\mathrm{CS}} / \Delta v=10^{13} \mathrm{~cm}^{-2}\left(\mathrm{~km} \mathrm{~s}^{-1}\right)^{-1}, N_{13} \mathrm{CO} / \Delta v=10^{16} \mathrm{~cm}^{-2}\left(\mathrm{~km} \mathrm{~s}^{-1}\right)^{-1}, x_{\mathrm{e}}=10^{-5}$; dash-dot-dotted line: $n_{\mathrm{H}}=100 \mathrm{~cm}{ }^{-3}$, $N_{\mathrm{CS}} / \Delta v=10^{14} \mathrm{~cm}^{-2}\left(\mathrm{~km} \mathrm{~s}^{-1}\right)^{-1}, N_{13} \mathrm{CO} / \Delta v=10^{16} \mathrm{~cm}^{-2}\left(\mathrm{~km} \mathrm{~s}^{-1}\right)^{-1}, x_{\mathrm{e}}=10^{-5}$. Kinetic temperatures increase in steps of $20 \mathrm{~K}$ in the direction of increasing CS $(J=2-1) T_{\mathrm{MB}}$.

of coupled differential equations for the CS rotation ladder

$$
\begin{aligned}
\frac{\mathrm{d} f_{i}}{\mathrm{~d} t}= & -\left\{\sum_{j<i}\left[C_{i j}+A_{i j} \beta_{i j}\left(1+J_{v_{i j}}\left(T_{\mathrm{bg}}\right)\right)\right]+\sum_{j>i}\left[C_{i j}\right.\right. \\
& \left.\left.+A_{j i} \beta_{j i} \frac{g_{j}}{g_{i}} J_{v_{j i}}\left(T_{\mathrm{bg}}\right)\right]\right\} f_{i}+\left\{\sum _ { j > i } f _ { j } \left[A_{j i} \beta_{j i}\left(1+J_{v_{j i}}\left(T_{\mathrm{bg}}\right)\right)\right.\right. \\
& \left.\left.+C_{j i}\right]+\sum_{j<i} f_{j}\left[C_{j i}+A_{i j} \beta_{i j} \frac{g_{i}}{g_{j}} J_{v_{i j}}\left(T_{\mathrm{bg}}\right)\right]\right\}
\end{aligned}
$$

where $f_{i}$ is the fractional population of the $i$ th energy level, subjected to the normalization condition $\sum_{i} f_{i}=1, A_{i j}$ is the Einstein coefficient for spontaneous emission (Chandra et al. 1995), $\beta_{i j}$ the escape probability (Neufel \& Melnick 1987), $C_{i j}$ the collision rate for the $i \rightarrow j$ transition (Turner et al. 1992; Turner 1996; Lique et al. 2006), $g_{i}=2 \times J_{i}+1$ the statistical weight of $i$ th rotational level, and

$$
J_{v_{i j}}\left(T_{\mathrm{bg}}\right)=\frac{1}{\exp \left(h v_{i j} / k T_{\mathrm{bg}}\right)-1}
$$

the background source at $T_{\mathrm{bg}}=2.7 \mathrm{~K}$. Collision partners include electrons (cf. Turner 1996). In the calculation we assume that the molecular hydrogen fraction is $n_{\mathrm{H}_{2}} / n_{\mathrm{H}} \gtrsim 0.3$, the ortho-para ratio in $\mathrm{H}_{2}$ is 3 , and $n_{\mathrm{He}} / n_{\mathrm{H}}=0.1$.

The (LVG) solution of the radiative transfer equation gives the main-beam brightness temperature

$T_{\mathrm{MB}}\left(v_{i j}\right)=\frac{h v_{i j}}{k}\left[J_{v_{i j}}\left(T_{\mathrm{ex}}\right)-J_{v_{i j}}\left(T_{\mathrm{bg}}\right)\right] \times\left(1-\mathrm{e}^{-\tau_{i j}}\right)$

where $T_{\mathrm{ex}}$ is the excitation temperature defined by the relation

$J_{v_{i j}}\left(T_{\mathrm{ex}}\right)=\frac{1}{f_{j} g_{i} / f_{i} g_{j}-1}=\frac{1}{\exp \left(h v_{i j} / k T_{\mathrm{ex}}\right)-1}$

and $\tau_{i j}$ the optical depth given by

$\tau_{i j}=\frac{c^{3}}{8 \pi v_{i j}^{3}} A_{i j}\left(f_{j} \frac{g_{i}}{g_{j}}-f_{i}\right) N_{\mathrm{CS}} / \Delta v$.

In Eq. (5) $N_{\mathrm{CS}} / \Delta v$ is the CS column density per unit velocity.
Using the equilibrium solution to the system (1) we derive the main-beam brightness temperature, Eq. (3), and the corresponding excitation temperature, obtained from Eq. (4), for gas densities ranging from $n_{\mathrm{H}}=50$ to $10000 \mathrm{~cm}^{-3}$, kinetic temperatures $T_{\mathrm{k}}=20$ to $100 \mathrm{~K}$, ionization fractions $x_{\mathrm{e}}=10^{-5}, 10^{-4}$, and CS column densities $N_{\mathrm{CS}} / \Delta v=$ $10^{11}, 10^{12}, 10^{13}, 10^{14} \mathrm{~cm}^{-2}\left(\mathrm{~km} \mathrm{~s}^{-1}\right)^{-1}$. Results are shown in Fig. 1.

The observed main-beam brightness temperature of core $B$ is reproduced for CS column densities larger than $10^{12} \mathrm{~cm}^{-2}\left(\mathrm{~km} \mathrm{~s}^{-1}\right)^{-1}$. To constrain both gas density and kinetic temperature we exploited the observations of ${ }^{13} \mathrm{CO}(J=1-0)$ emission from core $B$ reported in Scappini et al. (2000), that derived a (peak) main-beam brightness temperature $T_{\mathrm{MB}}=6.68(12) \mathrm{K}$ at $11.1 \mathrm{~km} \mathrm{~s}^{-1}$. Solving system (1), with suitable collision (Flower 2001; Balakrishnan et al. 2002; Cecchi-Pestellini et al. 2002) and radiative (Chandra et al. 1996) rates for ${ }^{13} \mathrm{CO}$, we computed main-beam brightness temperatures for the same set of physical and chemical models used in CS calculations. The assumed ${ }^{13} \mathrm{CO}$ column densities are in the range $N_{13} \mathrm{CO} / \Delta v=10^{13}-10^{18} \mathrm{~cm}^{-2}\left(\mathrm{~km} \mathrm{~s}^{-1}\right)^{-1}$. Results are reported in Fig. $3 \mathrm{a}$ and b, where we compare ${ }^{13} \mathrm{CO}(J=1-0)$ and CS $(J=2-1)$ main-beam brightness temperatures under a variety of conditions typical of diffuse and translucent clouds. Observed values are reproduced using $\mathrm{CS}$ and ${ }^{13} \mathrm{CO}$ column densities of $10^{13}$ and $10^{16} \mathrm{~cm}^{-2}\left(\mathrm{~km} \mathrm{~s}^{-1}\right)^{-1}$, respectively. Gas density and kinetic temperature are roughly $n_{\mathrm{H}} \lesssim 1000 \mathrm{~cm}^{-3}$ and $T_{\mathrm{k}}=20-30 \mathrm{~K}$. The electron fraction is $x_{\mathrm{e}} \sim 10^{-4}$. Using the empirical correlation between ${ }^{13} \mathrm{CO}$ column density and visual extinction derived by Hayakawa et al. (2001), we obtain $A_{\mathrm{V}}=4.2-6.1 \mathrm{mag}$.

In core $A$ we derived only the upper limit $T_{\mathrm{MB}}=$ 12(2) $\mathrm{mK}$, that constrains CS column density to be $N_{\mathrm{CS}} \lesssim$ $10^{13} \mathrm{~cm}^{-2}\left(\mathrm{~km} \mathrm{~s}^{-1}\right)^{-1}$. However, gas density and kinetic temperature are not well determined, since, almost all values in our parameter set produce consistent results. Using ${ }^{13} \mathrm{CO}(J=1-0)$, $T_{\mathrm{MB}} \sim 1 \mathrm{~K}$ (Scappini et al. 2002), and $\mathrm{CO}(J=2-1), T_{\mathrm{MB}} \sim$ $7.1 \mathrm{~K}$ (Scappini et al. 2000), data at $12.3 \mathrm{~km} \mathrm{~s}^{-1}$, we derive $N_{{ }^{13} \mathrm{CO}} / \Delta v=10^{15}$ and $N_{\mathrm{CO}} / \Delta v=10^{16} \mathrm{~cm}^{-2}\left(\mathrm{~km} \mathrm{~s}^{-1}\right)^{-1}, n_{\mathrm{H}} \lesssim$ $700 \mathrm{~cm}^{-3}, T_{\mathrm{k}}=20-30 \mathrm{~K}$, and $A_{\mathrm{V}} \lesssim 1 \mathrm{mag}$. Is is important to note that gas density decreases with increasing atomic hydrogen fraction, reaching values as low as $n_{\mathrm{H}}=100 \mathrm{~cm}^{-3}$. In this case, 
the kinetic temperature needed for the excitation of $\mathrm{CO}$ rotational ladder approaches $T_{\mathrm{k}}=100 \mathrm{~K}$. As shown by Liszt (2006), this behaviour is essentially due the effect of the $\mathrm{CO}-\mathrm{H}$ collisions, whose rates recently computed by Balakrishnan et al. (2002) imply a drastic revision of the $\mathrm{CO}$ excitation scheme.

Whatever is the atomic hydrogen content in core $A,{ }^{13} \mathrm{CO}$ column density does not vary significantly, as well as $A_{\mathrm{V}}$, according to the empirical correlation of Hayakawa et al. (2001). As a consequence core $A$ appears to be more "diffuse" than core $B$, consistently with our non-detection of CS $(J=$ 2-1) emission.

\section{Conclusions}

Chemical models predict that the concentrations of CS depend on dust extinction. This dependence probes the different cloud physical conditions: from diffuse to dense where radiative processes become gradually unimportant (Turner 1996). Previous ${ }^{13} \mathrm{CO}$ observations suggest that core $A$ is much more illuminated by the background UV field than core $B$. Present statistical equilibrium calculations on ${ }^{13} \mathrm{CO}$ and $\mathrm{CO}$ rotational ladders imply that core $A$ is less dense and hotter than core $B$. Thus, our observations in cores $A$ and $B$ are consistent with the general observational scenario for CS $(J=2-1)$ emission.

In the context of the present observations, an important point is related to the morphology of the region as seen at $\sim 11-12 \mathrm{~km} \mathrm{~s}^{-1}$ (Casu et al. 2005), and in particular the possibility that cores $A, B$ and $C$ belong to a single cloud complex. Scappini et al. (2002) suggested that, in spite of the low angular resolution of their observations, it is not unreasonable to suppose that these cores are physically related in the foreground of the stars Cyg OB2 Nos. 5 and 12. Their conclusions were also supported by $\mathrm{C}_{2}$ absorption measurements of Gredel \& Münch (1994). Nevertheless, the kinematic structure of the region appears to be very intricate, as evidenced by the presence of asymmetry in the ${ }^{13} \mathrm{CO}(J=1-0)$ line profiles (Casu et al. 2005) suggesting the existence of two or more layers of slightly different velocities along the line of sight. The velocity component at $11.1 \mathrm{~km} \mathrm{~s}^{-1}$ appears confined only in core $B$. On the contrary, the $12.3 \mathrm{~km} \mathrm{~s}^{-1}$ component is present in all cores $A, B$, and $C$. This component is also seen in core $B$ as a shoulder in the line profile centred at $11.1 \mathrm{~km} \mathrm{~s}^{-1}$.

Moreover, the intensity of the line at $11.1 \mathrm{~km} \mathrm{~s}^{-1}$ decreases smoothly with increasing distance from the emission peak, while the intensity of the $12.3 \mathrm{~km} \mathrm{~s}^{-1}$ component appears to be more erratic (McCall et al. 2002; Casu et al. 2005). We conclude that it is not likely that core $B$ belongs to the diffuse region observed at $12.3 \mathrm{~km} \mathrm{~s}^{-1}$. Indeed, core $B$ has morphology, chemistry and excitation conditions characteristic of translucent clouds, and could be a separate object similar to those listed in the Clemens \& Barvainis (1988) catalogue: a Bok globule.

\section{References}

Balakrishnan, N., Yan, M., \& Dalgarno, A. 2002, ApJ, 568, 443

Casu, S., Scappini, F., Cecchi-Pestellini, C., \& Olberg, M. 2005, MNRAS, 359, 73

Cecchi-Pestellini, C., \& Dalgarno, A. 2000, MNRAS, 313, L6

Cecchi-Pestellini, C., Bodo, E., Balakrishnan, N., \& Dalgarno, A. 2002, ApJ, 571,1015

Chandra, S., Kegel, W. H., Le Roy, R. J., \& Hertenstein, T. 1995, A\&AS, 114, 175

Chandra, S., Maheshwari, V. U., \& Sharma, A. K. 1996, A\&AS, 117, 557

Clemens, D. P., \& Barvainis, R. 1988, ApJS, 68, 257

Drdla, K., Knapp, G. R., \& van Dishoeck, E. F. 1989, ApJ, 345, 815

Flower, D. R. 2001, J. Phys. B, 34, 2731

Geballe, T. R., \& Oka, T. 1996, Nature, 384, 334

Gredel, R., \& Münch, G. 1994, A\&A, 285, 640

Gredel, R., van Dishoeck, E., \& Black, J. H. 1994, A\&A, 285, 300

Gredel, R., Black, J. H., \& Yan, M. 2001, A\&A, 375, 553

Hayakawa, T., Cambrésy, L., Onishi, T., Mizuno, A., \& Fukui, Y. 2001, PASJ, 53,1109

Heithausen, A. 2002, A\&A, 393, L41

Henning, Th., \& Launhardt, R. 1998, A\&A, 338, 223

Kokooline, V., Greene, C. H., \& Esry, B. D. 2001, Nature, 412, 891

Kopp, M., Gerin, M., Roueff, E., \& Le Bourlot, J. 1996, A\&A, 305, 558

Le Petit, F., Roueff, E., \& Herbst, E. 2004, A\&A, 417, 993

Lique, S., Spielfiedel, A., \& Chernicharo, J. 2006, A\&A, 451, 1125

Liszt, H. S. 2006, A\&A, 458, 507

Liszt, H. S., \& Lucas, R. 2001, A\&A, 370, 576

Liszt, H. S., \& Lucas, R. 2002, A\&A, 391, 693

Lucas, R., \& Liszt, H. S. 1996, A\&A, 307, 237

Lucas, R., \& Liszt, H. S. 2000, A\&A, 355, 327

Lucas, R., \& Liszt, H. S. 2002, A\&A, 384, 1054

McCall, B. J., Geballe, T. R., Hinkle, K. H., \& Oka, T. 1998, Science, 279, 1910

McCall, B. J., Hinkle, K. H., Geballe, T. R., et al. 2002, ApJ, 567, 391

McCall, B. J., Huneycutt, A. J., Saykally, R. J., et al. 2003, Nature, 422, 500

Neufeld, D. A., \& Melnick, G. J. 1987, ApJ, 322, 266

Scappini, F., Cecchi-Pestellini, C., Codella, C., \& Dalgarno, A. 2000, MNRAS, 317, L6

Scappini, F., Casu, S., Cecchi-Pestellini, C., \& Olberg, M. 2002, MNRAS, 337, 495

Smith, A. M., Lyu, C. H., \& Bruhweiler, F. C. 2001, BAAS, 33, 1452

Snow, T. P. 1976, ApJ, 204, L127

Turner, B. E., Chan, K.-W., Green, S., \& Lubowich, D. A. 1992, ApJ, 399, 114

Turner, B. E. 1996, ApJ, 461, 246 